\title{
Pseudoscalar correlators and the problem of the chiral limit in the compact lattice QED with Wilson fermions * $\dagger$
}

\author{
A. Hoferichter, V.K. Mitrjushkin ${ }^{\ddagger}$ and M. Müller-Preussker \\ Institut für Physik, Humboldt-Universität, 10099 Berlin, Germany
}

The phase diagram for the compact lattice QED with Wilson fermions is shown. We discuss different methods for the calculation of the 'pion' mass $m_{\pi}$ near the chiral transition point $\kappa_{c}(\beta)$.

\section{Phase diagrams}

The standard Wilson lattice action $S_{W A}$ for $4 d$ compact $U(1)$ gauge theory (QED) is

$S_{W A}=\beta \cdot S_{G}(U)+S_{F}(U, \bar{\psi}, \psi)$,

where $S_{G}(U)$ is the plaquette (Wilson) action for the pure gauge $U(1)$ theory, and

$S_{F}=\bar{\psi} \mathcal{M} \psi \equiv \bar{\psi}[\hat{1}-\kappa \cdot Q(U)] \psi$

is the fermionic part of the action.

In our calculations we also used the modified (compact) action $S_{M A}$ with Wilson fermions

$S_{M A}=S_{W A}(U)+\delta S_{G}(U)$,

where the additional term $\delta S_{G}$ is introduced to suppress some lattice artifacts, i.e., monopoles and negative plaquettes.

The phase diagram for the standard compact theory (with $S_{W A}$ ) is shown in Fig.1a [1] (the preliminary variant was in [2]). The line from $\left(\beta_{1} ; \kappa_{1}\right)$ to $\left(\beta_{2} ; \kappa_{2}\right)$ was found to be a $1^{\text {st }}$ order transition line which is driven by monopole condensation in the confinement phase. The order of the phase boundary between the confinement and the $4^{\text {th }}$ phase (i.e. the transition line from $\left(\beta_{2} ; \kappa_{2}\right)$ to $(\beta=0 ; 1 / 4))$ still remains open. A straightforward way to tackle this problem seems impossible, since standard fermionic bulk observables like

*TALK GIVEN AT THE LATTICE '94 INTERNATIONAL SYMPOSIUM LATTICE FIELD THEORY, BIELEFELD, GERMANY, SEPTEMBER 27 - OCTOBER 1, 1994

${ }^{\dagger}$ Work supported by the Deutsche Forschungsgemeinschaft under research grant $\mathrm{Mu} 932 / 1-3$

‡Permanent adress: JINR, Dubna, Russia $\langle\bar{\psi} \psi\rangle$ are ill-defined due to permanent occurence of near-to-zero eigenvalues of the fermionic matrix in the $4^{\text {th }}$ phase.

For $\kappa<\kappa_{1}$ and $\beta \simeq \beta_{0}$ the same phase transition as in the pure gauge theory is observed $\left(\beta_{0} \simeq 1\right.$ denotes the critical copling in the pure gauge theory). Time histories of the plaquette exhibit metastable states, which are probably due to monopole loops wrapping around the torus whereas no metastabilities occur for $\bar{\psi} \psi$. Therefore we cannot establish a $1^{\text {st }}$ order phase transition in this part of the phase diagram.

The 'horizontal' line from $\left(\beta_{1} ; \kappa_{1}\right)$ to $(\beta=$ $\left.\infty ; \kappa_{c}=1 / 8\right)$ which separates the Coulomb phase from the $3^{\text {rd }}$ phase corresponds to the chiral transition in this theory and is presumably a higher order phase transition line. For determining the chiral transition the variance of the pion norm $\sigma^{2}(\Pi)$ turned out to be a sensitive parameter :

$\sigma^{2}(\Pi) \sim V \cdot \frac{1}{N} \sum_{i}^{N}\left(\Pi_{i}-\bar{\Pi}\right)^{2}, \quad \bar{\Pi} \equiv \frac{1}{N} \sum_{i}^{N} \Pi_{i}$.

where for the $i^{\text {th }}$ configuration the value of $\Pi_{i}$ is given by $1 / 4 V \cdot \sum_{x y} \operatorname{Sp}\left(\mathcal{M}_{x y}^{-1} \gamma_{5} \mathcal{M}_{y x}^{-1} \gamma_{5}\right), V$ is the number of sites, and $N$ is the number of measurements. (Sp denotes the trace with respect to Dirac-indices). Near the chiral transition $\sigma^{2}(\Pi)$ seems to exhibit the characteristic features of a finite size scaling behaviour of a singular point on finite volume systems.

In case of the modified theory (eq.(3)) the situation drastically changes in comparison with the standard theory (see Fig.1b). Just two phases separated by a 'horizontal' chiral transition line $\kappa_{c}(\beta)$ survive. There is no sign for a $1^{\text {st }}$ order 


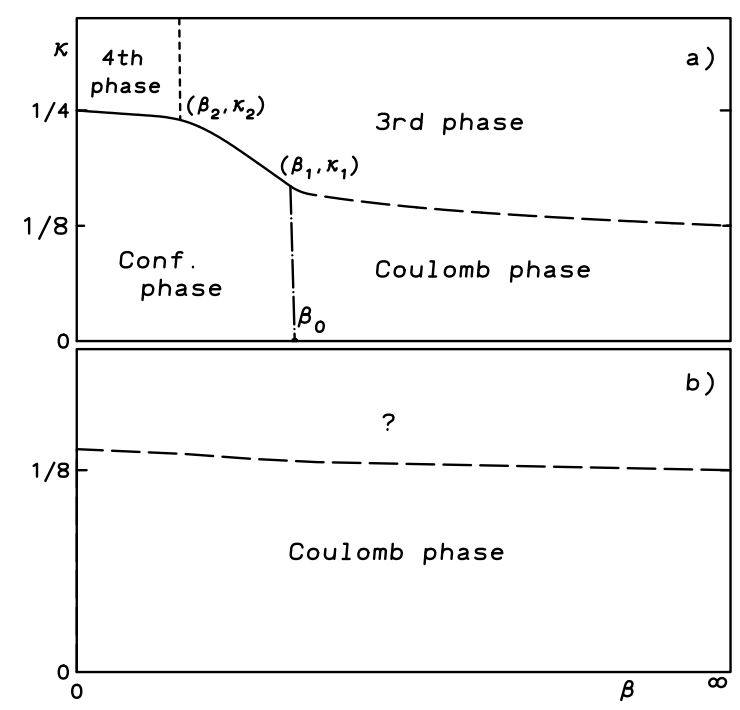

Figure 1. Phase diagram in the $(\beta, \kappa)$-plane for dynamical fermions for the standard Wilson action WA (a) and for the modified action MA (b) valid for $\kappa \lesssim 0.3$.

phase transition even at strong coupling. The average plaquette and the fermionic condensate, respectively, behave smoothly with varying $\kappa$ at fixed $\beta$, no metastabilities are observed. For greater detail and more discussion on the phase structure of both theories see [1].

\section{2. 'Pion' mass near $\kappa_{c}$}

The standard choice of the estimator for the effective mass of the pseudoscalar particle (for simplicity we'll call it the effective 'pion' mass) $m_{\pi}^{\text {eff }}(\tau) \equiv m_{\pi}(\tau)$ is

$m_{\pi}(\tau)=-\ln \frac{\hat{\Gamma}(\tau+1)}{\hat{\Gamma}(\tau)} ; \quad\left(\right.$ at $\left.\quad N_{\tau}=\infty\right)$

where $\hat{\Gamma}(\tau)$ is the pseudoscalar (zero-momentum) correlator

$\hat{\Gamma}(\tau)=\frac{1}{N} \cdot \sum_{i=1}^{N} \Gamma_{i}(\tau)$,

At large enough $\tau$ the dependence of $m_{\pi}(\tau)$ on $\tau$ should disappear (at least, in the case of the mass gap), and the resulting plateau gives the true mass $m_{\pi}$.

We will consider the approach to the chiral limit, i.e. $\kappa \rightarrow \kappa_{c}(\beta)$ in the confinement phase $\left(\beta<\beta_{0}\right)$ of the quenched standard theory. The well-known problem in QED and QCD with the calculation of $m_{\pi}$ (and other observables) is connected with extremely small eigenvalues of the fermionic matrix $\mathcal{M}$ which appear at $\kappa \sim \kappa_{c}(\beta)$. In Figs.2a,b the values of $\Gamma_{i}(\tau)$ are shown on individual configurations $i=1, . ., 500$ which form the correlator corresponding to eq. (6). The data are obtained from a $12 \times 4^{3}$ lattice at $\beta=0$ and $\kappa=1 / 4$ in the quenched approximation. The huge spikes of magnitude $\sim 10^{4}$ appearing on some configurations make the calculation of the mass $m_{\pi}$ in eq.(5) unreliable with the statistics at hand $\left(\sim 10^{2} \div 10^{4}\right.$ measurements $)$.

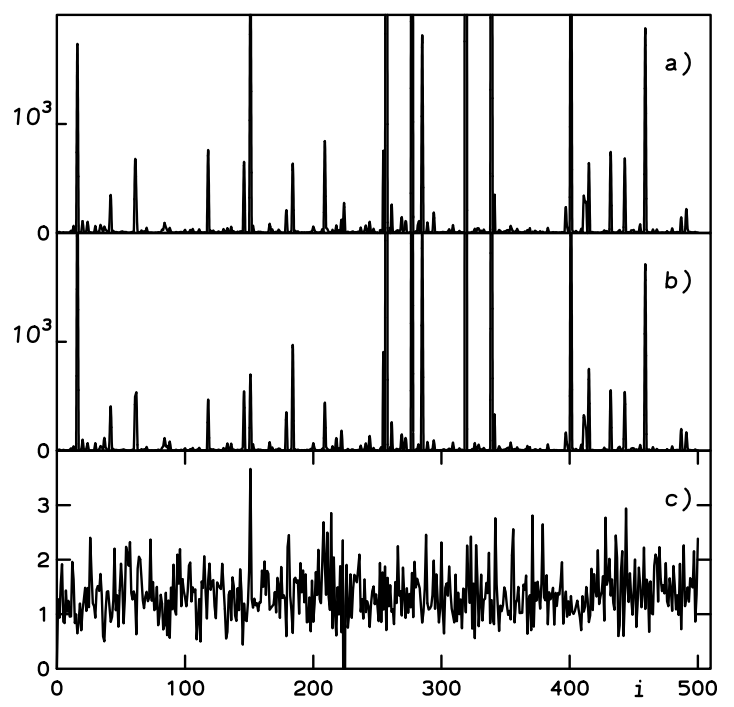

Figure 2. 'Time'-histories for pseudoscalar correlators $\Gamma_{i}(\tau)$ at $\tau=1(\mathbf{a}), \tau=2(\mathbf{b})$ and their ratio $(\mathbf{c}) . \kappa=0.25$ and $\beta=0$.

The usual way to avoid this problem is to decrease $\kappa$ which entails however the increasing of the mass (or $m_{\pi} / m_{\rho}$ ) up to unphysical values.

A possible way to extract reliable values of $m_{\pi}$ near the chiral transition point is based on the following observation. The 'time'-histories for the ratios $e^{-\mu_{i}(\tau)} \equiv \Gamma_{i}(\tau+1) / \Gamma_{i}(\tau)$ do not show such 
big fluctuations as the $\Gamma_{i}(\tau)$ 's themselves (see Fig.2c). Representing the correlator $\Gamma_{i}(\tau)$ in the form $\Gamma_{i}(\tau) \equiv \gamma_{i} \cdot f_{i}(\tau)$, one can conclude that for every $i^{\text {th }}$ configuration the contribution of the near-to-zero eigenvalues producing the peaks is factorised out in the $\tau$-independent factor $\gamma_{i}$. On the contrary, $e^{-\mu_{i}}$ or $\mu_{i}$ are statistically very well defined even at $\kappa^{\lambda} \kappa_{c}$.

From eq.(5) one can obtain

$e^{-m_{\pi}(\tau)}=e^{-m_{\pi}^{\prime}(\tau)}+\frac{1}{N} \sum_{i=1}^{N} e^{-\mu_{i}(\tau)} \cdot \frac{\delta \Gamma_{i}(\tau)}{\hat{\Gamma}(\tau)}$

where

$e^{-m_{\pi}^{\prime}(\tau)} \equiv \frac{1}{N} \sum_{i=1}^{N} e^{-\mu_{i}(\tau)}$

with $\delta \Gamma_{i}(\tau) \equiv \Gamma_{i}(\tau)-\hat{\Gamma}(\tau)$ and $\sum_{i}^{N} \delta \Gamma_{i}(\tau) \equiv 0$. The second term in the r.h.s in eq.(7) is supposed (for $N \rightarrow \infty$ ) to give a small correction to the first term because of the alternating factor $\delta \Gamma_{i}(\tau)$ in the sum. We checked it calculating $m_{\pi}(\tau)$ and $m_{\pi}^{\prime}(\tau)$ at small enough values of $\kappa<\kappa_{c}$ where the standard definition in eq.(5) works well. In this case both definitions give the same value of the pion mass. In Fig. 3 we show the behaviour of both estimators $m_{\pi}(\tau=5)$ and $m_{\pi}^{\prime}(\tau=5)$ as a function of $\kappa$ at $\beta=0$.

The 'pion' mass $m_{\pi}^{\prime}(\tau)$ shown in Fig.3 gradually decreases to some small value with increasing $\kappa$. Note, that for $\kappa$ 's 'far enough' from $\kappa_{c}$ both estimators agree within good accuracy. Preliminary, the transition seems to be a smooth one (at least at $\beta=0$ ), while the behaviour of the 'standard' mass $m_{\pi}(\tau)$ would not encourage to any conclusion. A similar picture holds for the cases $\beta=0.1$ and $\beta=0.8$ but here work is still in progress 沟.

\section{Conclusions}

We have studied the phase structure of two theories with Wilson fermions and compact action with $U(1)$ symmetry : standard Wilson theory and a modified one with lattice artifacts suppressed. Phase diagrams of both theories were shown.

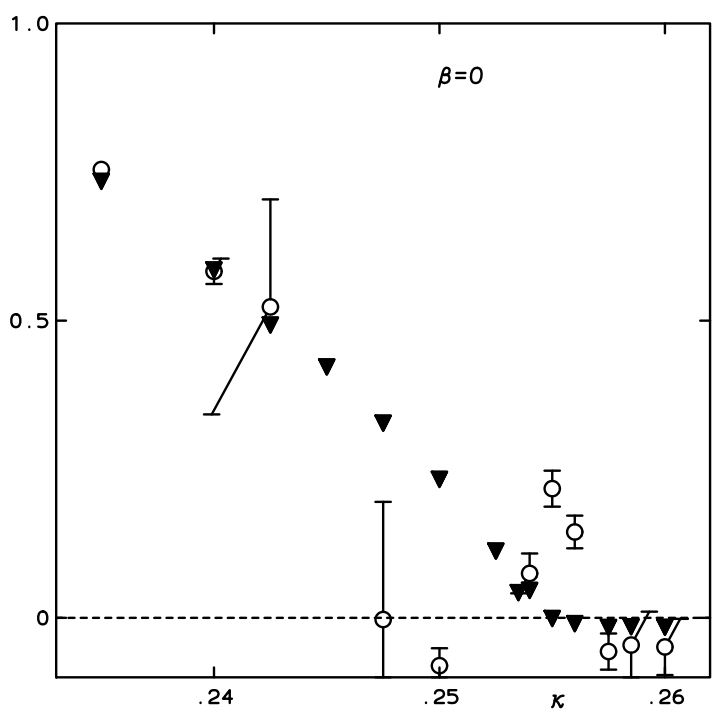

Figure 3. $m_{\pi}(\tau=5)$ (circles) and $m_{\pi}^{\prime}(\tau=5)$ (triangles) as a function of $\kappa$ at $\beta=0$ on a $12 \times 4^{3}$ lattice

When suppressing lattice artifacts the phase diagram changes strongly, and there is no universality in the strong coupling region. This resembles the situation with staggered fermions [3].

We propose another method for the calculation of the 'pion' mass near the chiral transition point $\kappa_{\sim} \gtrsim \kappa_{c}(\beta)$ which hopefully solves the well-known problem arising from near-to-zero eigenvalues of $\mathcal{M}$ in this $\kappa$-region.

The behaviour of the new estimator $m_{\pi}^{\prime}(\tau)$ should now be studied in the dynamical fermion case.

\section{REFERENCES}

1. A. Hoferichter, V. K. Mitrjushkin, M. MüllerPreussker, Th.Neuhaus and H.Stüben, DESY preprint-94/151 and HU Berlin-IEP-94/13, to be published in Nucl.Phys. B.

2. A.Hoferichter, V.K.Mitrjushkin, M.MüllerPreussker and Th.Neuhaus, Nucl.Phys.B (Proc.Suppl.) 34 (1994) 537.

3. A.Hoferichter, V.K.Mitrjushkin and M.Müller-Preussker, Phys.Lett. B 338 (1994) 325.

4. A.Hoferichter, V.K.Mitrjushkin and M.Müller-Preussker, in preparation 